\title{
Positive immunohistochemical staining of KIT in solid-pseudopapillary neoplasms of the pancreas is not associated with KITIPDGFRA mutations
}

\author{
Dengfeng $\mathrm{Cao}^{1}$, Cristina Antonescu ${ }^{2}$, Grace Wong ${ }^{2}$, Jordan Winter ${ }^{3}$, Anirban Maitra ${ }^{1,4}$, \\ N Volkan Adsay ${ }^{5}$, David S Klimstra ${ }^{2}$ and Ralph H Hruban ${ }^{1,4}$ \\ ${ }^{1}$ Department of Pathology, The Sol Goldman Pancreatic Cancer Research Center, The Johns Hopkins Medical \\ Institutions, Baltimore, MD, USA; ${ }^{2}$ Department of Pathology, Memorial Sloan-Kettering Cancer Center, \\ New York, NY, USA; ${ }^{3}$ Department of Surgery, The Sol Goldman Pancreatic Cancer Research Center, \\ The Johns Hopkins Medical Institutions, Baltimore, MD, USA ${ }^{4}$ Department of Oncology, The Sol \\ Goldman Pancreatic Cancer Research Center, The Johns Hopkins Medical Institutions, Baltimore, \\ MD, USA and ${ }^{5}$ Department of Pathology, Harper University Hospital, Detroit, MI, USA
}

Solid-pseudopapillary neoplasms of the pancreas are uncommon neoplasms of low malignant potential and of uncertain histogenesis. A small percentage of patients develop metastatic disease and some succumb to disease. The management of patients with metastatic disease or unresectable tumor, and patients who are just not good surgical candidates is problematic. Novel therapy targets are needed. Successful treatment of metastatic and unresectable gastrointestinal stromal tumors with KIT kinase inhibitor, imatinib mesylate (Gleevec), makes it intriguing to look at the status of $K I T$ in solid-pseudopapillary neoplasms of the pancreas. In this study, we investigated KIT expression in $\mathbf{5 0}$ solid-pseudopapillary neoplasms by immunohistochemical staining. Of the $50(50 \%)$ solid-pseudopapillary neoplasms, 25 showed diffuse expression (in $>50 \%$ neoplastic cells) of KIT and additional five (10\%) cases showed focal staining (in $10-50 \%$ neoplastic cells). Expression of KIT was not associated with tumor behavior and prognosis. A subset of 11 cases showing diffuse KIT expression detected by immunohistochemical staining were further evaluated for the presence of activating mutations in KIT exons 9, 11, 13 and 17, and PDGFRA exons 12 and 18 using PCR amplification followed by direct sequencing. However, no KIT or PDGFRA mutations were identified in any of these 11 cases tested, suggesting that the overexpression of KIT is probably not due to activating mutations in KIT or PDGFRA. The exact mechanism of KIT overexpression in solid-pseudopapillary neoplasms remains to be elucidated. One possible mechanism is gene dose effect (increased copies of KIT gene). Experience in gastrointestinal stromal tumors and other tumors have shown that mutation-mediated activation of KIT or PDGFRA is a prerequisite for clinical response with imatinib mesylate. Thus, lack of mutations in KIT or PDGFRA in solid-pseudopapillary neoplasms suggests that imatinib mesylate is less likely to be effective in the treatment for patients with metastatic disease or unresectable tumor, and patients who are just not good surgical candidates.

Modern Pathology (2006) 19, 1157-1163. doi:10.1038/modpathol.3800647; published online 16 June 2006

Keywords: solid-pseudopapillary neoplasm; pancreas; KIT; immunohistochemistry; molecular analysis; mutation

Solid-pseudopapillary neoplasms of the pancreas are uncommon neoplasms and of unknown histogenesis. ${ }^{1}$ Complete surgical resection has been

Correspondence: Dr D Cao, MD, PhD, Division of Surgical Pathology, Department of Pathology, The Johns Hopkins Hospital, Weinberg 2242, 401 North Broadway Street, Baltimore, MD 21231, USA.

E-mail: dcao1@jhmi.edu

Received 10 April 2006; revised and accepted 17 May 2006; published online 16 June 2006 reported to provide a $>95 \%$ cure rate. ${ }^{2}$ However, $10-15 \%$ of surgically resected tumors recur or develop metastasis ${ }^{2,3}$ and about $1-2 \%$ patients will succumb to disease. ${ }^{2,4}$ In addition, solid-pseudopapillary neoplasms rarely undergo sarcomatoid transformation that is invariably associated with a highly aggressive behavior. ${ }^{3}$ The management of patients with metastatic disease or unresectable tumor, patients whose tumor undergoes sarcomatoid transformation, and patients who are just not good surgical candidates is problematic. Novel therapeutic targets are needed for these patients. 
The discovery of imatinib mesylate (Gleevec) marks a new era of targeted therapy. Imatinib mesylate is a selective inhibitor of certain tyrosine kinases including KIT, platelet growth factor receptor alpha (PDGFRA) and BCR-ABL tyrosine kinases, etc. ${ }^{5}$ The human KIT gene was mapped on chromosome $4 \mathrm{q} 12$ and consists of 21 exons. ${ }^{6}$ The encoded protein KIT is a transmembrane protein belonging to the family of type III receptor tyrosine kinases that also includes PDGFRA. ${ }^{7,8}$ KIT plays an important role in the development of hematopoietic stem cells, mast cells, melanocytes, germ cells and interstitial cells of Cajal., ${ }^{9,10}$ The KIT protein is normally activated through binding to its ligand (stem cell factor). ${ }^{7,8}$ The neoplasms corresponding to the above cell types, such as chronic myeloproliferative disorders, acute myeloid leukemia, mastocytosis/ mast cell neoplasms, seminoma/dysgerminoma and gastrointestinal stromal tumors, often harbor KIT activating mutations that result in constitutive ligand-independent KIT phosphorylation and downstream activation. ${ }^{11}$ It has been found that $75-80 \%$ gastrointestinal stromal tumors harbor KIT activating mutations, which mainly cluster in 4 exons: 9, 11, 13 and $17 .{ }^{12-14}$ In addition, approximately one-third of gastrointestinal stromal tumors that lack KIT gene mutations harbor PDGFRA mutations (mainly in exons 12 and 18, rarely exon 14). ${ }^{15,16}$ Several studies have shown that imatinib mesylate is highly effective in treating chronic myelogenous leukemia ${ }^{17}$ and gastrointestinal stromal tumors ${ }^{18}$ but the efficacy in the latter is strongly associated with the types of activating mutations in KIT and PDGFRA. ${ }^{19,20}$

The clinical efficacy of imatinib mesylate in the treatment of chronic myelogenous leukemia and gastrointestinal stromal tumors has triggered investigation in a number of tumors lacking effective nonsurgical therapy. The aim of this study is to examine KIT expression in a large series of 50 solid-pseudopapillary neoplasms of the pancreas by immunohistochemical staining and correlate KIT expression with various clinicopathologic factors. We further evaluated the presence of activating mutations in KIT exons 9, 11, 13 and 17 and PDGFRA exons 12 and 18 investigated in a subset of 11 cases with diffuse immunohistochemical KIT expression.

\section{Materials and methods}

The permission was obtained from the Johns Hopkins Institutional Review Board to perform this study. In total, 50 histologically confirmed solidpseudopapillary neoplasms of the pancreas from the surgical pathology files of the Johns Hopkins Hospital, Harper University Hospital and Memorial Sloan-Kettering Cancer Center were included for this study. Of these 50 cases, 27 were reported in a previous study. ${ }^{3}$ The diagnostic criteria defined by World Health Organization $2000^{21}$ were used to histologically classify a pancreatic tumor as a solidpseudopapillary neoplasm: a combination of solid and cystic components with degenerative changes resulting in pseudopapillary formation, and uniform and polygonal epithelioid cells arranged in a discohesive pattern. For some cases, immunohistochemical stains with CD10 and/or $\beta$-catenin were also performed to further support the histological diagnosis. The clinicopathologic information was obtained through medical records and/or tumor registry.

\section{Immunohistochemical Staining}

Unstained $4 \mu \mathrm{m}$ sections were cut from each paraffin block and deparaffinized by routine techniques before placing in $200 \mathrm{ml}$ Target Retrieval Solution, pH 6.0 (Dako, Envision Plus Detection Kit, Carpinteria, CA, USA) for $20 \mathrm{~min}$ at $100^{\circ} \mathrm{C}$. After cooling for $20 \mathrm{~min}$, slides were quenched with $3 \% \mathrm{H}_{2} \mathrm{O}_{2}$ for $5 \mathrm{~min}$, before incubating with monoclonal antibody to KIT (CD117) (prediluted polyclonal antibody, Catalog CMA768, Cell Marque Inc., Hot Springs, AR, USA) for $60 \mathrm{~min}$ using the Dako Autostainer. Labeling was detected with the Dako Envision system as per the manufacturer's protocol. All sections were counterstained with Giles' hematoxylin. Immunolabeling of at least $10 \%$ neoplastic cells (cytoplasmic and/or membranous) was considered positive, and positive cases were further divided into focally positive (10-50\% cells) and diffusely positive ( $>50 \%$ cells).

\section{KIT and PDGFA Mutational Analysis}

A subset of 11 cases showing diffuse KIT expression by immunohistochemical staining were further evaluated for the presence of activating mutations in the KIT (exons 9, 11, 13 and 17) and PDGFRA (exons 12 and 18). Genomic DNA was isolated from formalin-fixed paraffin embedded tissue using the DNeasy $^{\mathrm{TM}}$ Tissue Kit (Qiagen Inc., Valencia, CA, USA). Of genomic DNA, $1 \mu \mathrm{g}$ was subjected to PCR using Platinum TaqDNA Polymerase High Fidelity (Life Technologies Inc., Gaithersburg, MD, USA). The PCR conditions were as follows: (1) $94^{\circ} \mathrm{C}$ for $4 \mathrm{~min}$; (2) $94^{\circ} \mathrm{C}$ for $30 \mathrm{~s}$, (3) the relevant annealing temperature for each primer set for $30 \mathrm{~s}$, (4) $72^{\circ} \mathrm{C}$ for $30 \mathrm{~s}$, (35 cycles) and (5) $72^{\circ} \mathrm{C}$ for $3 \mathrm{~min}$. The PCR products were identified by agarose gel electrophoresis using a $2 \%$ MetaPhor $^{\text {TM }}$ agarose gel (BioWhittaker Applications, Rockland, ME, USA). The PCR products were purified with the QIAquick ${ }^{\mathrm{TM}}$ PCR Purification Kit (Qiagen Inc., Valencia, CA, USA) before sequencing. The sequencing reactions for each case were performed from both the forward and reverse directions, using a $300 \mathrm{nM}$ PCR primer. All 11 cases were tested for KIT exons 9, 11, 13 and 17 as well as for PDGFRA exons 12 and 18. The 
primer sequences and annealing temperatures are listed in Table 1. Every ABI sequence was compared to a NCBI Human KIT and PDGFRA gene nucleotide sequence and blasted using a NCBI Standard Nucleotide Blast Search to determine the presence of mutation within a particular exon.

\section{Results}

In normal pancreatic tissue, only scattered interstitial cells of Cajal of the pancreas, ${ }^{10}$ mast cells, occasional acinar cells and rare cells within ductal epithelium are labeled with the antibody to KIT (Figure 1). In contrast, 25 of the 50 (50\%) solidpseudopapillary neoplasms of the pancreas showed diffuse KIT expression (in $>50 \%$ neoplastic cells) in the neoplastic cells (Figure 1). Additional five cases $(10 \%)$ showed focal KIT labeling (10-50\% tumor cells). The staining is mostly cytoplasmic with some membrane accentuation.

We further analyzed correlation between KIT expression and clinicopathologic factors (Table 2). Both groups (with and without KIT expression) were predominantly or all females (25 of 30 patients with KIT expression were females and all 20 patients without KIT expression were females) $(P=0.10)$. Patients with KIT expression were slightly older than those without ( 37 vs 33 years, $P=0.453$ ). Local invasion or extrapancreatic invasion was seen in five of 30 patients (one with extension into adrenal gland, one into duodenal wall, one into omentum, two into deep peripancreatic soft tissue) with and three of 20 patients (one into duodenal wall, two into deep peripancreatic soft tissue) without KIT expression $(P>0.05)$. Only rarely vascular

Table 1 KIT exons 9, 11, 13 and 17 and PDGFRA exons 12 and 18 primer sequences, with the corresponding annealing temperatures

\begin{tabular}{lllc}
\hline Exon \# & Primers & Primer sequences $5^{\prime} \rightarrow 3^{\prime}$ & $T_{A}\left({ }^{\circ} \mathrm{C}\right)$ \\
\hline $\begin{array}{l}\text { KIT } \\
9\end{array}$ & $\begin{array}{l}\text { Ex9-F } \\
\text { Ex9-R }\end{array}$ & $\begin{array}{l}\text { TTCCTAGAGTAAGCCAGGG } \\
\text { AATCATGACTGATATGGT }\end{array}$ & 53 \\
11 & Ex11-F & CCAGAGTGCTCTAATGACTG & 53 \\
& Ex11-R & ACCCAAAAAGGTGACATGGA & \\
13 & Ex13-F & ATCAGTTTGCCAGTTGTGCT & 53 \\
& Ex13-R & TTTATAATCTAGCATTGCC & \\
17 & Ex17-F & TGTGAACATCATTCAAGGCGTAC & 53 \\
& Ex17-R & CAGGACTGTCAAGCAGAGAATGG & \\
PDGFR $\alpha$ & & & \\
12 & Ex12-F & TCCAGTCACTGTGCTGCTTC & 54 \\
& Ex12-R & GCAAGGGAAAAGGGAGTCTT & \\
18 & Ex18F & ACCATGGATCAGCCAGTCTT & 55 \\
& Ex18R & TGAAGGAGGATGAGCCTGACC & \\
\hline
\end{tabular}

invasion was observed in both groups (three of 30 with KIT expression vs one of 20 without KIT expression, $P>0.05)$. Perineural invasion was observed in four of 30 tumors with KIT expression and three of 20 without KIT expression $(P>0.05)$. One of the 30 patients with KIT expression had metastasis in one regional lymph node, and no patient in the patients without KIT expression had node metastasis $(P>0.05)$. These findings indicate that there is no significant correlation between KIT expression and patient gender, age, local invasion (extrapancreatic invasion), vascular invasion and perineural invasion or lymph node metastasis.

Occasionally patients with solid-pseudopapillary neoplasms develop distant metastasis. ${ }^{3,4}$ Liver was the only distant metastasis site in patients in this study. Hepatic metastasis was seen in five of $30(17 \%)$ patients with KIT expression and two of $20(10 \%)$ patients without KIT expression $(P>0.05)$. Two of the five patients in the former group (expressing KIT) died of disease (Patient 1 (focal KIT expression), a 33-year-old female, had a liver metastasis when her pancreatic tumor was resected and this patient died 6 months later; Patient 2 (diffuse KIT expression), a 78-year-old female, had a liver metastasis 57 months later after her pancreatic tumor resection and died 3 months later after discovery of her liver metastasis). Patients 3 and 4 (both diffuse KIT expression, 20- and 56-year-old when diagnosed) in the former group had liver metastasis at the time of their initial pancreatic tumor resection and were alive at 40 and 217 months after hepatic metastasis resection. Patient 5 (diffuse KIT expression) was a 22-year-old female now alive with a liver metastasis 72 months later after her initial pancreatic tumor resection. Among the two patients with liver metastasis in the latter group (without KIT expression), patient 1 (a 56-year-old female) whose liver metastasis was found at the time of initial pancreatic tumor resection died of metastatic disease 115 months later, and patient 2 (a 41-yearold female) had liver metastasis 11 months later after her initial pancreatic tumor resection and now is still alive since hepatic metastasis was resected 121 months ago. Therefore, although in both groups a small percentage of patients developed distant metastasis (five of 30 patients with KIT and two of 30 patients without KIT expression) $(P>0.05)$ or even died of disease (two of 30 patients with KIT expression and one of 20 patients without KIT expression) $(P>0.05)$, KIT expression does not seem to correlate with liver metastasis and tumorassociated death.

Among the 25 cases showing diffuse KIT expression by immunohistochemical staining, a subset of 11 cases were further evaluated for the presence of mutations in KIT exons 9, 11, 13 and 17 as well as PDGFRA exons 12 and 18 using PCR-based amplification followed by direct sequencing. Among these 11 tumors, histologically four had at least one malignant feature. ${ }^{21}$ More specifically, all four cases 



Figure 1 Immunohistochemical stain of KIT in solid pseudopapillary neoplasms of the pancreas. Both pseudopapillary (a1) and solid (b1) areas express KIT (a2, b2). Sometimes the tumor shows vascular invasion (c1) and perineural invasion and those areas can also express KIT (c2, d1). In normal pancreas, only interstitial cells of Cajal of the pancreas (d2), mast cells, occasional acinar cells, and rare cells within the ductal epithelium label for this antigen. 
Table 2 Comparison of clinical and pathologic features between patients whose solid-pseudopapillary neoplasms had KIT expression and those did not

\begin{tabular}{|c|c|c|c|}
\hline $\begin{array}{l}\text { Clinical and } \\
\text { pathologic } \\
\text { features }\end{array}$ & $\begin{array}{l}\text { With KIT } \\
\text { expression } \\
(\mathrm{N}=30)\end{array}$ & $\begin{array}{l}\text { Without KIT } \\
\text { expression } \\
(\mathrm{N}=20)\end{array}$ & P-valu \\
\hline Female:Male & $25: 5$ & $20: 0$ & 0.10 \\
\hline $\begin{array}{l}\text { Age at diagnosis } \\
\text { (years) }\end{array}$ & $37 \pm 18(9-78)$ & $33 \pm 15(14-73)$ & 0.453 \\
\hline $\begin{array}{l}\text { Local invasion or } \\
\text { extrapancreatic } \\
\text { invasion }\end{array}$ & 5 of 30 & 3 of $20(15 \%)$ & $>0.05$ \\
\hline Vascular invasion & of 30 & 1 of 20 & $>0.05$ \\
\hline eri & 4 OI 3 & $15 \%)$ & $>0.05$ \\
\hline $\begin{array}{l}\text { Lymph node } \\
\text { metastasis }\end{array}$ & 1 of 30 & 0 of 20 & $>0.05$ \\
\hline $\begin{array}{l}\text { Cases demonstrating } \\
\text { at least one } \\
\text { malignant histo- } \\
\text { logical feature }^{\mathrm{a}}\end{array}$ & $10 / 30(33 \%)$ & 6 of $20 \%(30 \%)$ & $>0.05$ \\
\hline $\begin{array}{l}\text { Mean follow-up } \\
\text { (range) months }\end{array}$ & $52(3-217)$ & $52(2-121)$ & $>0.0$ \\
\hline Live & 5 of 3 & 2 of 2 & $>0.05$ \\
\hline Death due to disease & $2 / 30(7 \%)$ & $1 / 20(5 \%)$ & $>0.05$ \\
\hline
\end{tabular}

${ }^{\mathrm{a}}$ Local or extrapancreatic invasion, vascular invasion, perineural invasion, lymph node metastasis and liver metastasis.

had local invasion, two had vascular invasion, three had perineural invasion, one had lymph node metastasis, and one had liver metastasis. None of these 11 cases harbored a mutation in KIT exons 9, 11, 13 and 17 or in PDGFRA exons 12 and 18.

\section{Discussion}

In this study, we evaluated KIT expression in 50 solid-pseudopapillary neoplasms of the pancreas with immunohistochemical staining. Our results show that KIT is expressed in a significant subset (30 of 50 cases or $60 \%, 25$ diffuse and five focal) of solid-pseudopapillary neoplasms of the pancreas. However, there was no significant correlation between KIT expression and clinicopathologic factors such as age, gender, extrapancreatic tumor extension or local invasion, vascular invasion, perineural invasion, lymph node metastasis, distant or liver metastasis, or disease-specific death.

We further evaluated the presence of activating mutations in the KIT and PDGFRA genes in 11 solidpseudopapillary neoplasms of the pancreas that demonstrated diffuse immunohistochemical expression of the KIT protein. Some of these 11 tumors demonstrated local aggressive behavior (local invasion, vascular invasion, perineural invasion), node metastasis and/or distant metastasis. None of these 11 tumors analyzed harbored activating mutations in KIT and PDGFRA genes, suggesting that the KIT overexpression in solid-pseudopapillary neoplasms of the pancreas is probably not due to activating mutations in KIT or PDGFRA. Although we did not sequence all the tumors expressing diffuse KIT by immunohistochemical staining, lack of association between KIT expression and clinicopathologic factors AND lack of KIT/PDGFRA mutations in all 11 tumors analyzed (among which some demonstrated local aggressive behavior, node metastasis and/or distant metastasis) suggests that either KIT expression or KIT/PDGFRA mutation is probably not associated with tumor behavior.

The molecular mechanism of KIT overexpression in solid-pseudopapillary neoplasms of the pancreas remains unknown. Immunohistochemical staining of KIT has been demonstrated in many types of tumors that do not or only very rarely harbor KIT or PDGFRA activating mutations. These tumors include adenoid cystic carcinomas of the salivary gland, ${ }^{22,23}$ pulmonary small cell carcinomas, ${ }^{24,25}$ breast carcinomas, ${ }^{26}$ chromophobe renal cell carcinomas and oncocytomas ${ }^{27}$ serous ovarian carcinomas, ${ }^{28}$ melanomas, ${ }^{29,30}$ angiosarcomas, ${ }^{11}$ and neural tumors such as glioblastomas and oligodendrogliomas. $^{31}$ The absence of KIT or PDGFRA activating mutations in these neoplasms and solid-pseudopapillary neoplasms that express KIT detected by immunohistochemical staining suggests that there is (are) mechanism(s) other than activating mutations to overexpress or upregulate KIT protein. One possible mechanism is gene dose effect as a result of increased copies of KIT gene. Recently, Sihto et $a 1^{24}$ have demonstrated that seven of 12 (including two glioblastomas, one medulloblastoma, one clear cell renal cell carcinoma, three pulmonary small cell carcinomas) tumors showing strong KIT expression by immunohistochemical staining had either KIT gene amplification or multiple copies of chromosome 4. It will be interesting to know whether KIT gene amplification and/or increased copies of chromosome 4 are responsible for KIT overexpression in solid-pseudopapillary neoplasms of the pancreas as well as in other tumors that do not harbor mutations but with immunohistochemcial expression of KIT.

Several studies have shown that in GISTs, clinical efficacy of imatinib mesylate is strongly associated with the types of activating mutations in KIT or PDGFRA genes..$^{15,19,20}$ As a result, it is not that surprising that multiple clinical trials have observed only minimal or no clinical efficacy of imatinib mesylate (Gleevec) in various types of tumors expressing KIT detected by immunohistochemical staining but lacking activating mutations, such as adenoid cystic carcinomas of the salivary gland, ${ }^{22}$ pulmonary small cell carcinomas, ${ }^{32,33}$ breast carcinomas, ${ }^{34}$ ovarian carcinomas $^{35}$ and melanomas. ${ }^{36}$ These studies ${ }^{15,19,20,22,32-36}$ support the concept that mutation-mediated activation of KIT or PDGFRA is a prerequisite for successful treatment with imatinib mesylate. ${ }^{37}$ This conclusion is further supported by a recent study in which clinical response to imatinib mesylate was observed in a patient with metastatic thymic carcinoma harboring an activating KIT mutation. ${ }^{38}$ In our study, we did not observe any KIT or 
PDGFRA mutations in all 11 analyzed cases with diffuse KIT expression detected by immunohistochemical staining making it less likely that patients with metastatic disease, unresectable disease, and those who are not good surgical candidates will benefit from imatinib mesylate treatment.

Although the morphologic features of solidpseudopapillary neoplasm of the pancreas have been well characterized, ${ }^{21}$ the histogenesis of this distinctive neoplasm remains to be determined. The phenotype of solid-pseudopapillary neoplasm of the pancreas does not resemble that of any of the normal epithelial counterparts of the pancreas. ${ }^{1}$ Recently, the interstitial cells of Cajal of the pancreas have been identified. ${ }^{10}$ These cells express KIT and have phenotypic characteristics of the interstitial cells of Cajal of the intestine. ${ }^{10}$ The finding of KIT expression in solid-pseudopapillary neoplasms suggests that they, at least at the level of KIT expression, show some differentiation towards the interstitial cells of Cajal of the pancreas. While this is intriguing, there is little other evidence to support such a hypothesis. First, CD34 expression is seen in two-thirds of gastrointestinal stromal tumors $^{39}$ but not in solid-pseudopapillary neoplasms of the pancreas. ${ }^{1}$ Second, ultrastructural features of solid-pseudopapillary neoplasms of the pancreas differ from those of interstitial cells of Cajal of the pancreas. ${ }^{10,40}$ Third, a true gastrointestinal stromal tumor of the pancreas harboring KIT mutations has been reported. ${ }^{41}$ It is also interesting to note that the stromal cells of a pancreatic hamartoma also coexpressed KIT and CD34. ${ }^{42}$ Coexpression of KIT and CD34 is a feature of interstitial cell of Cajal of both the intestine and the pancreas. ${ }^{10}$

In summary, in this study we investigated KIT expression by immunohistochemical staining in 50 histologically confirmed solid-pseudopapillary neoplasms of the pancreas. We found that KIT expression is seen in a significant portion of these neoplasms (30 of 50 tumors or $60 \%$ ). However, KIT expression detected by immunohistochemical staining does not correlate with various clinicopathologic factors including tumor behavior. We did not identify any KIT (exons 9, 11, 13 and 17) or PDGFRA (exons 12 and 18) mutations in a subset of 11 cases showing diffuse KIT expression by immunohistochemical staining. Lack of mutations in KIT or PDGFRA in solid-pseudopapillary neoplasms of the pancreas suggests that imatinib mesylate is less likely to be effective in the treatment for patients with metastatic disease or unresectable tumor, and patients who are just not good surgical candidates.

\section{References}

1 Kosmahl M, Seada LS, Janig U, et al. Solid-pseudopapillary tumor of the pancreas: its origin revisited. Virchows Arch 2000;436:473-480.
2 Klimstra DS, Wenig BM, Heffess CS. Solid-pseudopapillary tumor of the pancreas: a typically cystic carcinoma of low malignant potential. Semin Diagn Pathol 2000;17:66-80.

3 Tang LH, Aydin H, Brennan MF, et al. Clinically aggressive solid pseudopapillary tumors of the pancreas: a report of two cases with components of undifferentiated carcinoma and a comparative clinicopathologic analysis of 34 conventional cases. Am J Surg Pathol 2005;29:512-519.

4 Papavramidis T, Papavramidis S. Solid pseudopapillary tumors of the pancreas: review of 718 patients reported in English literature. J Am Coll Surg 2005;200: 965-972.

5 Capdeville R, Buchdunger E, Zimmermann J, et al. Glivec (STI571, imatinib), a rationally developed, targeted anticancer drug. Nat Rev Drug Discov 2002;1: 493-502.

6 Yarden Y, Kuang WJ, Yang-Feng T, et al. Human protooncogene KIT: a new cell surface receptor tyrosine kinase for an unidentified ligand. EMBO J 1987;6: 3341-3351.

7 Pawson T. Regulation and targets of receptor tyrosine kinases. Eur J Cancer 2002;38:S3-S10.

8 Vanderback GR, deCastro CM, Taylor H, et al. Cloning and structural analysis of the human KIT gene. Oncogene 1992;7:1259-1266.

9 Gibson PC, Cooper K. CD117 (KIT): a diverse protein with selective applications in surgical pathology. Adv Anat Pathol 2002;9:65-69.

10 Popescu LM, Hinescu ME, Ionescu N, et al. Interstitial cells of Cajal in pancreas. J Cell Mol Med 2005;9: 169-190.

11 Miettinen S, Sarlomo-Rikala M, Lasota J. KIT expression in angiosarcomas and fetal endothelial cells: lack of mutations of exon 11 and exon 17 of KIT. Mod Pathol 2000;13:536-541.

12 Hirota S, Isozaki K, Moriyama Y, et al. Gain-of-function mutations of KIT in human gastrointestinal stromal tumors. Science 1998;279:577-580.

13 Lux ML, Rubin BP, Biase TL, et al. KIT extracellular and kinase domain mutations in gastrointestinal stromal tumors. Am J Pathol 2000;156:791-795.

14 Rubin BP, Singer S, Tsao C, et al. KIT activation is a ubiquitous feature of gastrointestinal stromal tumors. Cancer Res 2001;61:8118-8121.

15 Corless CL, Schroeder A, Griffin D, et al. PDGFRA mutations in gastrointestinal stromal tumors: frequency, spectrum and in vitro sensitivity to imatinib. J Clin Oncol 2005;23:5357-5364.

16 Heinrich MC, Corless CL, Duensing A, et al. PDGFRA activating mutations in gastrointestinal stromal tumors. Science 2003;299:708-710.

17 Druker BJ, Talpaz M, Resta DJ, et al. Efficacy and safety of a specific inhibitor of the BCR-ABL tyrosine kinase in chronic myeloid leukemia. N Engl J Med 2001;344: 1031-1037.

18 Demetri GD, von Mehren M, Blanke CD, et al. Efficacy and safety of imatinib mesylate in advanced gastrointestinal stromal tumors. N Engl J Med 2002;347: $472-480$.

19 Debiec-Rychter M, Dumez H, Judson I, et al. Use of KIT/PDGFRA mutational analysis to predict the clinical response to imatinib in patients with advanced gastrointestinal stromal tumours entered on phase I and II studies of the EORTC Soft Tissue and Bone Sarcoma Group. Eur J Cancer 2004;40:689-695. 
20 Heinrich MC, Corless CL, Demetri GD, et al. Kinase mutations and imatinib response in patients with metastatic gastrointestinal stromal tumor. J Clin Oncol 2003;21:4342-4349.

21 Hamilton SR, Aaltonen LA. Pathology and Genetics Tumors of the Digestive System. World Health Organization Classification of Tumors: Lyon, 2000.

22 Hotte SJ, Winquist EW, Lamont E, et al. Imatinib mesylate in patients with adenoid cystic cancers of the salivary glands expressing KIT: a Princess Margaret Hospital phase II consortium study. J Clin Oncol 2005; 23:585-590.

23 Holst VA, Marshall CE, Moskaluk CA, et al. KIT protein expression and analysis of KIT gene mutation in adenoid cystic carcinoma. Mod Pathol 1999;12: 956-960.

24 Sihto H, Sarlomo-Rikala M, Tynninen O, et al. KIT and platelet-derived growth factor receptor alpha tyrosine kinase gene mutations and KIT amplifications in human solid tumors. J Clin Oncol 2005;23:49-57.

25 Tamborini E, Bonadiman L, Negri T, et al. Detection of overexpressed and phosphorylated wild-type kit receptor in surgical specimens of small cell lung cancer. Clin Cancer Res 2004;10:8214-8219.

26 Simon R, Panussis S, Maurer R, et al. KIT (CD117)positive breast cancers are infrequent and lack KIT gene mutations. Clin Cancer Res 2004;10:178-183.

27 Haitel A, Susani M, Wick N, et al. KIT overexpression in chromophobe renal cell carcinoma is not associated with KIT mutation of exons 9 and 11. Am J Surg Pathol 2005;29:842.

28 Lassus H, Sihto H, Leminen A, et al. Genetic alterations and protein expression of KIT and PDGFRA in serous ovarian carcinoma. Br J Cancer 2004;91: 2048-2055.

29 Pache M, Glatz K, Bosch D, et al. Sequence analysis and high-throughput immunohistochemical profiling of KIT (CD 117) expression in uveal melanoma using tissue microarrays. Virchows Arch 2003;443:741-744.

30 Went PT, Dirnhofer S, Bundi M, et al. Prevalence of KIT expression in human tumors. J Clin Oncol 2004;22: 4514-4522.

31 Burger H, den Bakker MA, Kros JM, et al. Activating mutations in KIT and PDGFRa are exclusively found in gastrointestinal stromal tumors and not in other tumors overexpressing these imatinib mesylate target genes. Cancer Biol Ther 2005;4:1270-1274.

32 Dy GK, Miller AA, Mandrekar SJ, et al. A phase II trial of imatinib (ST1571) in patients with KIT expressing relapsed small-cell lung cancer: a CALGB and NCCTG study. Ann Oncol 2005;16:1811-1816.

33 Krug LM, Crapanzano JP, Azzoli CG, et al. Imatinib mesylate lacks activity in small cell lung carcinoma expressing KIT protein: a phase II clinical trial. Cancer 2005;103:2128-2131.

34 Modi S, Seideman AD, Dickler M, et al. A phase II trial of imatinib mesylate monotherapy in patients with metastatic breast cancer. Breast Cancer Res Treat 2005; 90:157-163.

35 Coleman RL, Broaddus RR, Bodurka DC, et al. Phase II trial of imatinib mesylate in patients with recurrent platinum- and taxane-resistant epithelial ovarian and primary peritoneal cancers. Gynecol Oncol 2006;101: 126-131.

36 Ugurel S, Hildenbrand R, Zimpfer A, et al. Lack of clinical efficacy of imatinib in metastatic melanoma. Br J Cancer 2005;92:1398-1405.

37 von Mehren M. Targeted therapy with imatinib: hits and misses? J Clin Oncol 2005;23:8-10.

38 Strobel P, Hartmann M, Jakob A, et al. Thymic carcinoma with overexpression of mutated KIT and the response to imatinib. $N$ Engl J Med 2004;350: 2625-2626.

39 Fletcher CD, Berman JJ, Corless C, et al. Diagnosis of gastrointestinal stromal tumors: a consensus approach. Hum Pathol 2002;33:459-465.

40 Matsunou H, Konishi F. Papillary-cystic neoplasm of the pancreas. A clinicopathologic study concerning the tumor aging and malignancy of nine cases. Cancer 1990;65:283-291.

41 Daum O, Klecka J, Ferda J, et al. Gastrointestinal stromal tumor of the pancreas: case report with documentation of KIT gene mutation. Virchows Arch 2005; 446:470-472.

42 Pauser U, da Silva MT, Placke J, et al. Cellular hamartoma resembling gastrointestinal stromal tumor: a solid tumor of the pancreas expressing KIT (CD117). Mod Pathol 2005;18:1211-1216. 